\title{
Title: Dental Pulp Stem Cells Scope in Dentistry; A Review
}

\author{
Dr. C Pushpalatha, Dr.Anand Nimbal, Dr.Shrenik Jain And \\ Dr.Praveen Tammannavar \\ Bharati Vidyapeeth Deemed University Dental College And Hospital, Wanlesswadi,Sangli-416414, \\ Maharashtra.
}

\begin{abstract}
Dental pulp is a well known tissue enrich of adult mesenchymal stem cells called Dental Pulp Stem cells (DPSc). These adult stem cells play an important role in regenerative medicine both for oral and non oral pathoses with biological properties such as multipotency, high proliferation rates and accessibility. Dental pulp stem cells were primarily derived from the pulp tissues of exfoliated deciduous teeth, primary incisors and permanent third molar teeth. Role of stem cells for hard tissue formation has considerably increased attention of researchers as these cells can be a fascinating source of stable differentiated cells, capable of inducing bone formation and control hydroxyapatite crystal growth. Dental professionals have the opportunity to make their patients aware of these new sources of stem cells that can be stored for future use as new therapies are developed for a range of diseases and injuries.
\end{abstract}

Keywords: Stem cells, dental pulp stem cells, SHED, Tissue engineering

Submitted date 12 June 2013

Accepted Date: 17 June 2013

\section{Introduction}

Stem cells are immature, undifferentiated cells that can divide and multiply for an extended period of time, differentiating into specific types of cells and tissues. Stem cells that reside in dental pulp, called dental pulp stem cells (DPSCs) are considered as a population of MSCs. Autogenous stem cells are derived from the patient being treated, while allogenous stem cells are derived from other individuals. Stem cells available commercially are mainly allogenous and will not produce an immune response. whereas, autogenous reduce the risk of rejection ${ }^{1}$. The process by which stem cells are derived from one type of tissue and differentiate into other types of tissue is referred to as plasticity or transdifferentiation ${ }^{2}$.

Stem cells can be divided into two categories - embryonic and adult. Embryonic stem cells are totipotent cells derived from the early mammalian embryo at the blastocyst stage able to differentiate into any cell type, as well as being propagated indefinitely in an undifferentiated state ${ }^{3}$. However, embryonic cells loose these properties as differentiation ensues and growth-promoting signals decline. Adult stem cells are not totipotent, and they can be further classified depending on their origin and differentiation potential ${ }^{3,4,5,6,7,8,9}$.

There are different population of stems cells like hematopoietic stem cells, bone marrow stromal cells and postnatal stem cells. Dental pulp stem cells represent a postnatal stem cells, capable of extensive proliferation and multipotential differentiation. The general properties of stem cells are unspecialized; continuous self-renewal; and can give rise to specialized cell types. Dental pulp stem cells are attractive for their potential to differentiate into several cell types including odontoblasts, neural progenitors, chondrocytes, endothelocytes, adipocytes, smooth muscle cells and osteoblasts. (Sloan \& Waddington, 2009) ${ }^{10}$.

\section{Sources of Dental pulp stem cells}

The first type of dental stem cell was isolated from the human pulp tissue and termed dental pulp stem cells (DPSCs) by Gronthos et al., 2000 based on their clonogenic abilities and rapid proliferative rates. In human postnatal dental tissue, five different sources of mesenchymal stem cells (MSCs) have been already identified: dental pulp, periodontal ligament, exfoliated deciduous teeth, dental follicle (DF), and root apical papilla. These dental stem cells are derived from the neural crest, and thus have a different origin from bone marrow-derived MSCs, which are derived from mesoderm. Among them, all except SHED are from permanent teeth ${ }^{11}$.

\section{Deciduous verus permanent dental pulp stem cells}

The dental pulp stem cells from the permanent third molar were first isolated by Gronthos et $\mathrm{al}^{12}$ who have described the cells as colonogenic cells capable of producing osteoblastic and chondrocytic cells in vitro and odontoblastic cells in vivo. Investigations have indicated the broad differentiation capacity of the pulp derived cells including their ability in giving rise to neural and endothelial cell lineages as well ${ }^{13,14,15}$. These 
cells may provide an appropriate source of specialized cells (odontoblast) for pulp regeneration in regenerative medicine.

Stem cells from deciduous incisor teeth were first investigated by Miura et $\mathrm{al}^{16}$ who found that an exfoliated human deciduous tooth contains multiopotent stem cells. These stem cells derived from human exfoliated deciduous teeth were called as SHED. SHED are immature, unspecialized cells in the teeth that are able to grow into specialized cell types by a process known as "differentiation." During the embryonic stage of human development i.e at the 6th week SHED is noticed. Dr. Songtao Shi in 2003, first discovered SHED. Abbas et al (2008) investigated the possible neural crest origin of dental pulp stem cells from exfoliated deciduous teeth (SHED) ${ }^{17}$.

The importance of stem cells derived from deciduous teeth is that they are derived from a tissue that is similar in some ways to the umbilical cord. These stem cells would be discarded, if not used. Investigations have indicated that SHED is originated from the neural crest, hence expressing neural markers. These cells have the ability to differentiate among neural cell lineages in addition to their potential of differentiating along odontoblastic, osteoblastic, chondrocytic and adipocytic cells ${ }^{18}$.

The primary teeth are a better source for therapeutic stem cells than wisdom teeth, and orthodontically extracted teeth. Recent studies have shown that SHED has the ability to develop into more types of body tissues than other types of stem cells. The cells derived from dental pulp cells have potential to differentiate into somatic cells including neurocytes, myocytes, adipocytes,chondrocytes and osteocytes ${ }^{17}$.

\section{Clinical applications}

Dental pulp is most feasible and rich source of mesenchymal stem cells: Dental Pulp Stem cells (DPSc) to be used in regenerative therapy. The physiologically intact dental pulp stem cells could be successfully differentiated to advanced derivatives of all three primary germlayers (odontoblast, osteoblast, chondrocyte, myocyte, neurocyte, adipocyte, corneal epithelial cell, melanoma cell,induced pluripotent stem cells iPSC) ${ }^{19}$.

Stem cell-based therapies are being investigated for the treatment of many conditions, including neurodegenerative conditions such as Parkinson's Disease and Multiple Sclerosis, liver disease, diabetes, cardiovascular disease, autoimmune diseases, musculoskeletal disorders and for nerve regeneration following brain or spinal cord injury. Currently, patients are being treated using stem cells for bone fractures, cancer (bone marrow transplants) and spinal fusion surgery. New stem cell therapies are continually under review, and some have already been approved by the U.S. Food and Drug Administration ${ }^{20}$. The comprehensive list of diseases and conditions currently being treated using stem cells include Stem Cell Disorders, Acute and chronic Leukemias, Myeloproliferative Disorders, Myelodysplastic Syndromes, Lymphoproliferative Disorders, Inherited Erythrocyte Abnormalities, Liposomal Storage Diseases, Histiocytic Disorders, Phagocyte Disorders, Congenital Immune System Disorders, Inherited Platelet Abnormalities, Plasma Cell Disorders and malignancies $^{21,22,23}$.

The applications related to oral health care have included regeneration of an immature tooth with extensive coronal and pulp damage, regeneration of resorbed root, cervical, or apical dentin, periodontal regeneration, whole-tooth regeneration, repair and replacement of bone in craniofacial defects(eg, repair of cleft lip/palate) can facilitate restoring the physiologic structural integrity. The successful regeneration of periodontal tissue, alveolar bone, cementum, and periodontal ligament has been achieved using autologous periodontal ligament mesenchymal stem cells (PDL-MSCs) ${ }^{24}$.

The regeneration of orodental tissues is dependent on four basic components. The appropriate signals, cells, blood supply, and scaffold that are needed to target the tissue at the site of defect. All these components are essential for reconstruction and healing of lost tissues. The cells provide the machinery for new tissue growth and differentiation, whereas the growth factors modulate the cellular activity and stimulate the cells to differentiate as well as produce tissue matrix. The new vascular tissues provide the nutritional base for tissue growth and the scaffolds guide and create a template structure in three-dimensions to facilitate the tissue regeneration process ${ }^{25}$.

\section{Stem Cell Handling and Cryopreservation}

Stem cells are released from small amounts of tissue, in the case of dental stem cells from dental pulp. The tissue is placed in an enzyme solution that releases the stem cells, which are then cultured to multiply. This can be accomplished using serum-free medium, removing the need for use of animal serum. Differentiation then occurs and the cells are transplanted - either alone or with a scaffold or other biomaterials, depending on the application.

Extracted permanent and deciduous (including exfoliating) teeth can be preserved for future use with cryopreservation. The cells are rapidly cooled to subzero temperatures as low as $-196^{\circ}$, stopping any cellular or biochemical activity. Rapid freezing is necessary to prevent ice from forming around or inside the cells and to prevent dehydration, as these would cause cell damage and death. Research has demonstrated that stem cells 
derived from the dental pulp of extracted third molars retain the ability to differentiate into multiple cell types following thawing after cryopreservation using liquid nitrogen. Stem cells derived from the periodontal ligament are viable following cryopreservation. After two years of cryopreservation, stem cells have been able to differentiate and to proliferate, and it has been concluded that DSCs can undergo long-term cryopreservation ${ }^{26}$, 27,28

\section{Conclusion}

Banking teeth and dental stem cells offers patients a viable alternative to using more invasive or ethically problematic sources of stem cells, and harvesting can be done during routine procedures in adults and from the deciduous teeth of children. The possibility of using stem cells, biological molecules and tissue engineering in clinical dentistry, opens completely new approaches to restore functionally and an aesthetically suitable tooth arch. Now, dental professionals have the opportunity to make their patients aware of these new sources of stem cells that can be conveniently recovered and remotely stored for future use as new therapies are developed for a range of diseases and injuries.

\section{References}

[1]. Barry FP, Murphy JM. Mesenchymal stem cells: clinical applications and biological characterization. Int J Biochem Cell Biol. 2004; 36 (4):568-84.

[2]. Ryu KH, Cho SJ, Jung YJ, Seoh JY, Kie JH, et al. In vitro generation of functional dendritic cells from human umbilical cord blood CD34+ cells by a 2-step culture method. Int J Hematol. 2004; 80: 281-6.

[3]. Paloma Dias Telles, Maria Aparecida de Andrade Moreira Machado, Vivien Thiemy Sakai, Jacques Eduardo Nor: Pulp tissue from primary teeth: new source of stem cells, J Appl Oral Sci. 2011;19(3):189-194

[4]. Ballini A, De Frenza G, Cantore S, Papa F, Grano M, Mastrangelo F, et al. In vitro stem cell cultures from human dental pulp and periodontal ligament: new prospects in dentistry. Int J Immunopathol Pharmacol. 2007; 20:9-16.

[5]. Edwards PC, Mason JM. Gene-enhanced tissue engineering for dental hard tissue regeneration: (2) dentin-pulp and periodontal regeneration. Head Face Med. 2006; 2:16.

[6]. Gronthos S, Zannettino A, Hay SJ, Shi S, Graves SE, Kortesidis A, et al. Molecular and cellular characterization of highly purified stromal stem cells derived from human bone marrow. J Cell Sci. 2003; 116:1827-35.

[7]. Huang GT, Shagramanova K, Chan S. Formation of odontoblastlike cells from cultured human dental pulp cells on dentin in vitro. J Endod. 2006; 32:1066-73.

[8]. Koyama N, Okubo Y, Nakao K, Bessho K. Evaluation of pluripotency in human dental pulp cells. J Oral Maxillofac. Surg. 2009; 67:501-6.

[9]. Maria OM, Khosravi R, Mezey E, Tran SD. Cells from bone marrow that evolve into oral tissues and their clinical applications. Oral Dis. 2006, 13:11-6.

[10]. Sloan, AJ. \& Waddington R. (2009) . Dental pulp stem cells: what, where, how? International Journal of Paediatric Dentistry. Vol. 19, No.1, (January 2009), pp.61-70.

[11]. Masaki J. Honda, Mari Imaizumi, Shuhei Tsuchiya and Christian Morsczeck: Dental follicle stem cells and tissue engineering, Journal of Oral Science, Vol. 52, No. 4, 541-552, 2010.

[12]. Gronthos S, Mankani M, Brahim J, Robey PG, Shi S. Postnatal human dental pulp stem cells (DPSCs) in vitro and in vivo. Proc Natl Acad Sci USA 2000 Dec 5;97(25):13625-30

[13]. Laino G, Carinci F, Graziano A, d'Aquino R, Lanza V, De Rosa A, et al. In vitro bone production using stem cells derived from human dentalpulp. J Craniofac Surg 2006 May; 17(3):511-5.

[14]. d'Aquino R, Graziano A, Sampaolesi M, Laino G, Pirozzi G, De Rosa A, et al. Human postnatal pulp cells co-differentiate into osteoblast and endotheliocytes: a pivotal synergy leading to adult bone tissue formation. Cell Death Differ 2007 Jun; 14(6): $1162-71$.

[15]. Zhang W, Walboomers XF, Shi S, Fan M, Jansen JA. Multilineage differentiation potential of stem cells derived from human dental pulp after cryopreservation. Tissue Eng 2006 Oct;12(10): 2813-23.

[16]. Miura M, Gronthos S, Zhao M, Lu B, Fisher LW, Robey PG, et al. SHED: stem cells from human exfoliated deciduous teeth. Proc Natl Acad Sci USA 2003 May 13; 100(10):5807-12.

[17]. Vipin Arora, Pooja Arora, AK Munshi Banking Stem Cells from Human Exfoliated Deciduous Teeth (SHED): Saving for the Future, J Clin Pediatr Dent 33(4): 289-294, 2009

[18]. MR. Baghaban Eslaminejad, S. Vahabi, M. Shariati, H. Nazarian, In vitro Growth and Characterization of Stem Cells from Human Dental Pulp of Deciduous Versus Permanent Teeth, Journal of Dentistry, Tehran University of Medical Sciences, Tehran, Iran (2010; Vol. 7, No.4)

[19]. Thekkeparambil Chandrabose Srijaya, Padmaja Jayaprasad Pradeep, RosnahBinti Zain, SabriMusa, NoorHayaty AbuKasim, and Vijayendran Govindasamy; The Promise of Human Induced Pluripotent StemCells in Dental Research; Stem Cells International, Volume 2012, 1-10

[20]. Jay B. Reznick. Continuing Education: Stem Cells: Emerging Medical and Dental Therapies for the Dental Professional. Dentaltown magazine,Oct: 42-53, 2008.

[21]. Arthur A, Rychkov G, Shi S, Koblar SA, Gronthos S. Adult human dental pulp stem cells differentiate toward functionally active neurons under appropriate environmental cues. Stem Cells, 26(7): 1787-95, 2008

[22]. Jeremy J. Mao. Stem Cells and the Future of Dental Care. New York State Dental Journal, 74(2): 21-24, 2008

[23]. Mao JJ, Giannobile WV, Helms JA, Hollister SJ, Krebsbach PH, Longaker MT, Shi S. Craniofacial tissue engineering by stem cells. J Dent Res, 85(11): 966-79, 2006.

[24]. R. R. Nadig, "Stem cell therapy-hype or hope? A review," Journal of Conservative Dentistry, vol. 12, no. 4, pp. 131-138, 2009.

[25]. S. Sood, S. Gupta, and A. Mahendra, "Gene therapy with growth factors for periodontal tissue engineering-a review," Medicina Oral, Patolog'ia Oral y Cirug' '́a Bucal, vol. 12, no. 2, pp. e301-e310, 2012.

[26]. Zhang W, Walboomers XF, Shi S, Fan M, Jansen JA. Multilineage differentiation potential of stem cells derived from human dental pulp after cryopreservation. Tissue Eng. 2006;12(10):2813-23.

[27]. Seo BM, Miura M, Sonoyama W, Coppe C, Stanyon R, Shi S. Recovery of stem cells from cryopreserved periodontal ligament. J Dent Res. 2005; 84(10):907-12. 
[28]. paccio G, Graziano A, d'Aquino R, Graziano MF, Pirozzi G, Menditti D, De Rosa A, Carinci F, Laino G. Long-term cryopreservation of dental pulp stem cells (SBP-DPSCs) and their differentiated osteoblasts: a cell source for tissue repair. J Cell Physiol. 2006; 208(2):319-25. 